Somnologie 2013 $\cdot 17: 122-130$ DOI 10.1007/s11818-013-0611-3

Eingegangen: 14. Januar 2013

Angenommen: 13. März 2013

Online publiziert: 6. Juni 2013

๑) Springer-Verlag Berlin Heidelberg 2013

\author{
A. Rodenbeck ${ }^{1,2}$ \\ ${ }^{1}$ CharitéCrossOver (CCO), Institut für Physiologie , Charité-Universitätsmedizin Berlin \\ ${ }^{2}$ Abteilung für Lungenheilkunde, Pneumologie und Schlaflabor, \\ Evangelisches Krankenhaus Weende gGmbH, Bovenden-Lenglern
}

\title{
Manual der American Academy of Sleep Medicine
}

\section{Überblick über die Updateversion 2.0}

Nachdem der Schlaf jahrtausendelang mehr oder weniger als rein passiver $\mathrm{Zu}$ stand verstanden wurde, wird er seit dem letzten Jahrhundert nunmehr als ein messtechnisch erfassbares Forschungsobjekt bzw. als Gegenstand für Diagnostik und Therapie von Schlaf-Wach-Störungen verstanden. Schulz stellt diese Entwicklung am Beispiel Berlin $[19,20]$ umfassend dar und gibt einen Überblick über die Entwicklung der Schlafstadienklassifikation [21]. Im Jahr 1968 veröffentlichte die Arbeitsgruppe um Rechtschaffen $u$. Kales [14] einen Vorschlag zur Erfassung und Auswertung verschiedener Schlafstadien, die auf der simultanen Ableitung von mindestens 2 Elektroenzephalogrammen (EEG, C3, C4), 2 Elektrookulogrammen (EOG, rechts, links), sowie auf einem 2-kanaligen mentalen oder submentalen Elektromyogramm (EMG) bei jungen Gesunden basierte. Dieses Rechtschaffenund-Kales(R\&K)-Manual - erstellt von hochkarätigen EEG-Spezialisten - etablierte sich innerhalb kürzester Zeit als der Goldstandard sowohl zur Erfassung als auch zur Auswertung von Schlaf.

Bei der Umsetzung dieses Standards von der rein wissenschaftlichen Ebene hin zur klinischen Anwendung bei SchlafWach-gestörten Patienten wurde jedoch schnell zum einen deutlich, dass das Spektrum der notwendigen abzuleitenden Kanäle um zumindest die im R\&K-Manual nicht enthaltenen Atmungs- und Beinbewegungsparameter erweitert werden musste. Zum anderen wurde offensichtlich, dass die Schlafstadienanalyse nach
Rechtschaffen und Kales bei Patienten nicht in allen Fällen angewendet werden kann bzw. einer gestörten Schlafphysiologie nur bedingt gerecht wird. Diese Kritikpunkte gibt es zwar ebenso lange wie das R\&K-Manual selbst, jedoch beschränkten sich bisherige Lösungsversuche auf die Definition entweder einzelner Ableitkanäle bzw. deren Auswertung oder auf die Definition einzelner elektrophysiologischer Ereignisse.

\section{Manualetablierung}

Im Jahr 2007 legte die American Academy of Sleep Medicine (AASM) nach fast 40 Jahren ein weiteres Manual vor [13]. Es wurden hierin die Ableittechniken und -kanäle und deren Auswerteregeln unter Berücksichtigung einer halbautomatischen computerisierten Auswertung in einer evidenzbasierten Vorgehensweise zusammenfassend dargestellt. Die alten R\&K-Regeln wurden - dort wo bisher nicht definiert - anpasst. Das R\&K-Manual beruhte auf einem Konsens der beteiligten Spezialisten und die Evaluation sollte erst anschließend erfolgen. Im Gegensatz hierzu basieren die Inhalte des AASM-Manuals mit empfohlenen, alternativen und optionalen Regeln auf einer evidenzbasierten Literaturanalyse. Dort, wo diese im Ergebnis nicht ausreicht, beruhen sie auf etablierten formalen Konsensusentscheidungen [12], sog. Research-and-development(RAND)-Verfahren, zur Bestimmung von Überversorgung, Unterversorgung und Angemessenheit medizinischer Prozeduren [9].
In der ersten AASM-Manualversion wurde eine regelmäßige Überprüfung und Überarbeitung angekündigt. Dies gilt umso mehr, da das AASM-Manual sich explizit nicht nur auf Gesunde bezieht, sondern ausdrücklich die Wertigkeit der Schlafmedizin betont. Die erste Version sollte mittlerweile aufgrund der autorisierten deutschen Übersetzung und entsprechender Übersichten [13, 17] gut bekannt sein. Aktuell liegt Version 2.0 des AASM-Manuals $[6,7]$ vor, in der v. a. die Regeln der Atmungserfassung und -auswertung überarbeitet und geändert wurden. Des Weiteren wurden häufige Nachfragen zu einzelnen Punkten eingearbeitet, die dem Internetportal ,frequently asked questions" (FAQ) des AASM-Manuals entnommen wurden.

\section{Wesentliche Änderungen}

Um die Änderungen ([15]) im Vergleich zur ersten AASM-Manualversion möglichst übersichtlich darzustellen, folgt die Auflistung der Manualstrukur. Nach einer kurzen Übersicht über die wesentlichen Punkte der ersten Version von 2007 werden die Veränderungen bzw. Klarstellungen angegeben. Es werden, wie in der ersten Version, Empfehlungen für die Routinemessung und -auswertung von Polysomnographien (PSG) gegeben. Alternativen werden ggf. benannt, die nach Ermessen des Arztes bzw. Untersuchers angewendet werden können. Auch sind sog. Optionen, d. h. Regeln für seltene Ereignisse mit unklarer physiologischer Bedeu- 
tung oder Ereignisse für die keine Konsusentscheidung getroffen werden konnte, enthalten. Sofern nicht anders angegeben, werden im Folgenden die Empfehlungen genannt. Aus dem Anhang des AASM-Manuals wird allerdings ersichtlich, dass die allermeisten Empfehlungen lediglich auf einem Konsens und nur wenige auf einer ausreichend hohen Evidenz beruhen.

\section{Technische Spezifikationen zur Polysomnographie}

In Version 2.0 bleiben die grundsätzlichen Festlegungen für PSG-Ableitungen unverändert. Dies betrifft im Wesentlichen die - definierte Epochendauer von 30 s und Vorgabe der PSG-Parameter, Ausstattung digitaler PSG-Geräte, PSG-Bildschirmanzeige und die digitale Auswertung von PSG-Daten;

- vorgegebenen Einstellungen für Filter und Empfindlichkeiten sowohl für die klassischen Schlafparameter als auch für die Atemkanäle (Atemfluss, Thorax- und Abdomenexkursionen, Sauerstoffsättigung usw.) und die Muskelaktivität der M. tibialis anterior;

- Vorgabe der Parameter, die im PSGBericht berechnet und angegeben werden sollen, wobei die Berechnungsweise vorgegeben ist;

- zwingende Darstellung der PSG-Geräte welche Epochen computerisiert und welche manuell ausgewertet wurden.

Die empfohlenen Abtastraten wurden für den endtidalen $\mathrm{pCO}_{2}$ und den Positive-airway-pressure(PAP)-Geräteatemfluss erweitert und entsprechen denen des nasalen Staudrucks $(100 \mathrm{~Hz}$, minimal $25 \mathrm{~Hz}$ ). Für die Filtereinstellungen finden sich nun auch Angaben für den PAP-Geräteatemfluss (DC) und den nasalen Staudruck (untere Grenzfrequenz: DC bzw. $\leq 0,03 \mathrm{~Hz}$; obere Grenzfrequenz: $100 \mathrm{~Hz}$ ).

\section{Polysomnographieparameter und Bericht}

Die folgenden Vorgaben für die PSG-Parameter und den PSG-Bericht wurden neu hinzugefügt, verändert oder präzisiert.
Das EKG ist nun auch als obligater Bestandteil einer PSG benannt; vermutlich wurde dies in der ersten Version übersehen. Es entfällt der Hinweis, dass Arrhythmien im PSG-Bericht nur noch angegeben werden müssen, sofern sie auftreten. Gefordert ist nun vielmehr immer eine Angabe zu den Parametern, die in der ersten AASM-Manualversion unter 1.E 4-10 (neue Listung in Version 2.0: 1.D 4-10) aufgeführt sind.

Die Anzahl der (berechneten) Parameter hinsichtlich der Atmungsereignisse wurde von 16 auf 25 erweitert und teils deutlich geändert. Im Wesentlichen ist die optionale Unterscheidung zwischen obstruktiven und zentralen Hypopnoen hinzugekommen. Im PSG-Bericht kann deren jeweilige Anzahl bzw. neben dem ApnoeHypopnoe-Index (AHI) auch ein obstruktiver und zentraler AHI optional angegeben werden. Hinzugefügt wurde als mögliche Angabe im Bericht auch das Auftreten von Schnarchen und der Respiratorydisturbance-Index (RDI). Dieser entspricht der Summe aus dem AHI und dem Respiratory-effort-related-arousal(RERA)-Index. Sofern bei einer PSG eine Kohlendioxidpartialdruck $\left(\mathrm{pCO}_{2}\right)$-Messung vorgenommen wird, muss der Bericht hierzu auch eine Angabe enthalten. Dies gilt sowohl für diagnostische PSGs als auch für PAP-Titrationen. Die empfohlenen Angaben zur Cheyne-Stokes-Atmung beschränken sich in Version 2.0 nicht nur auf die Angaben ja bzw. nein, sondern sind um die Angaben zur Dauer (absolut oder als Prozent der Gesamtschlafzeit) erweitert.

\section{Schlafstadienerfassung}

Neben der Einteilung der Schlafstadien nach Rechtschaffen und Kales erfolgt seit Jahrzehnten auch eine Einteilung in Nonrapid-eye-movement(Non-REM)- und REM-Schlaf. Das AASM-Manual aus dem Jahr 2007 machte diese Parallelität deutlich, indem die Stadien in Non-REM(N)1, N2 und N3 sowie REM (R) eingeteilt werden. Die folgenden grundlegenden Regelungen bleiben aus dem Jahr 2007 erhalten, die Präzisierungen sind kursiv gehalten.

\section{Elektroenzephalogramm}

Die Erfassung des EEGs erfolgt auf mindestens 3 Kanälen: neben der zentra- len Ableitung (C3) kommt eine frontale (F3) und eine okzipitale (O1) hinzu. Damit werden auf jeden Fall sowohl schlafrelevante $\delta$-Wellen (frontales Maximum) als auch $\alpha$-Wellen (okzipitale Betonung) und schlafspezifische Graphoelemente wie K-Komplexe (zentrale Betonung) am Ort ihrer jeweiligen maximalen Ausprägung gut erfasst. Zur Sicherheit sollen die Kanäle (Backup-Kanäle) $\mathrm{C} 4, \mathrm{~F} 4$ und $\mathrm{O} 2$ mit abgeleitet werden; die jeweilige Verschaltung auf das kontralaterale Mastoid verändert sich nicht. Als alternative Ableitung kann Fz-Cz, Cz-Oz, C4-M1 (mit Fpz, C3, O1, M2 als Backup-Kanäle) gewählt werden. In Version 2.0 wird deutlich gemacht, dass mit dieser alternativen EEG-Ableitung die $\delta$-Aktivität nicht adäquat erfasst werden kann. Lösungen dazu werden vorgegeben (siehe Stadium N3).

\section{Elektromyogramm}

Die standardisierte Erfassung des submentalen/mentalen EMGs erfolgt durch 3 Elektroden, wobei ein submentales EMG ( $2 \mathrm{~cm}$ unterhalb der Unterkieferkante und zwar $2 \mathrm{~cm}$ rechtsseitig bzw. linksseitig der Mittellinie) gegen ein mentales EMG (1 cm oberhalb der Unterkieferkante in der Mittellinie) abgeleitet wird. Das andere submentale EMG mit Verschaltung gegen dasselbe mentale EMG gilt als Reserve, wobei vorausgesetzt wird, dass eine Umschaltung von der einen zur anderen EMG-Elektrode während der laufenden Ableitung möglich ist.

\section{Elektrookulogramm}

Weiterhin ist neben der klassischen R\&KAbleitung der EOGs (1 cm unterhalb des linken äußeren Augenwinkels und $1 \mathrm{~cm}$ oberhalb des rechten äußeren Augenwinkels) alternativ auch eine Platzierung jeweils $1 \mathrm{~cm}$ unterhalb und seitlich des äußeren Augenwinkels möglich, wobei diese Elektroden dann jeweils gegen $\mathrm{Fp}_{z}$ abgeleitet werden sollen.

Die Schlafstadien werden in N1, N2, N3 und R (früher S1, S2, S3/S4 und REM) eingeteilt. Hierbei werden die ehemaligen Schlafstadien S3 und S4 als N3 zusammengefasst, wobei - wie schon im R\&KManual - altersunabhängig mindestens $20 \%$ tiefschlafrelevante $\delta$-Wellen $(0,5-$ 
Somnologie 2013 · 17:122-130 DOI 10.1007/s11818-013-0611-3

(c) Springer-Verlag Berlin Heidelberg 2013

\section{A. Rodenbeck \\ Manual der American Academy of Sleep Medicine. Überblick über die Updateversion 2.0}

\section{Zusammenfassung}

Im Jahr 2007 erschien das Manual der American Academy of Sleep Medicine (AASM), in dem erstmals alle notwendigen Parameter einer kardiorespiratorischen Polysomnographie (PSG) evidenzbasiert zusammenfassend mit technischen Spezifikationen, Ableite- und Auswertungsregeln dargestellt wurden. Eine regelmäßige Überprüfung und Überarbeitung dieser Regeln und Kriterien ist ein wesentlicher Bestandteil dieses Manuals. Aktuell liegt nun Version 2.0 vor; wesentliche Änderungen und deren möglichen Auswirkung auf die klinische Routine deutscher Labors werden dargestellt.

Die Änderungen betreffen vorwiegend die Erfassung und Auswertung der Atmungsparameter. So gibt es nur noch eine Definition der Hypopnoe mit einer 30\%igen Verminderung des Atemflusses über mindestens $10 \mathrm{~s}$ verbunden mit einem Abfall der
Sauerstoffsättigung um wenigstens 3\% oder einem Arousal. Auch sollen Apnoen gewertet werden, deren Beginn oder Ende in eine Epoche Wach fällt, sofern Schlaf folgt oder voran ging. Weiterhin kann nun die Erfassung sowohl von Apnoen als auch von Hypopnoen mittels oronasalem Thermistor oder Staudruckmessung erfolgen, wenn die jeweils andere Methode aus technischen Gründen nicht zur Verfügung steht. Des Weiteren gibt es Änderungen für die Definitionen und Erfassung von Hypoventilationen und der Cheyne-Stokes-Atmung. Im Bereich des Schlafsocrings wird kurz erläutert, dass - je nach Verwendung der empfohlenen oder alternativen Elektroenzephalogramm(EEG)und Elektrookulogramm(EOG)-MontageKombination ggf. das Stadium N3 auch über C4-M1 ermittelt werden kann.
Während v. a. die nunmehr einheitliche Hypopnoebewertungsregel und die Möglichkeit, Apnoen und Hypopnoen mittels nur einer Methode zu erfassen, für die klinische Routine eine große Erleichterung sein dürften, müssen sich andere Vorgaben erst noch in der Praxis bewähren. Spätestens mit Version 2.0 des AASM-Manuals stellt sich auch die Frage, ob die Vorgaben des Manuals als Ganzes in Deutschland verpflichtend werden sollen oder ob zumindest bestimmte Grundprinzipien auch für ältere PSG-Geräte umsetzbar sind. Für wissenschaftliche Fragestellungen ist eine vollständige Umsetzung des AASM-Manuals in der Version 2.0 unabdingbar.

Schlüsselwörter

Schlaf $\cdot$ Atmung $\cdot$ Scoringmethoden .

Polysomnographie $\cdot$ Equipement

\section{Manual of the American Academy of Sleep Medicine. Review of the update version 2.0}

\section{Abstract}

In the 2007 manual of the American Academy of Sleep Medicine (AASM), all parameters for a cardiorespiratory polysomnography (PSG) were published for the first time in an evidence-based manner including technical specifications and rules for derivations and scoring. After the regularly planned review and revision, the current version 2.0 was published. The significant modifications and their possible impact on clinical routine in Germany are discussed.

The most important changes are related to the measurement and scoring of the parameters of breathing. Only one definition remains for the scoring of hypopneas with a flow decrease of $30 \%$ over at least $10 \mathrm{~s}$ associated with either a desaturation of at least $3 \%$ or an arousal. In addition, apneas should be rated if they start or end in an epoch scored as wake whenever the patient showed sleep before or afterwards. The revised version allows the measurement of both apneas and hypopneas using an oronasal thermistor or a nasal pressure transducer in the case the other method is not possible due to technical reasons. Furthermore, the definitions and scoring of hypoventilation and CheyneStokes breathing have changed. Concerning sleep scoring, it should be noted that stage N3 can also be determined using C4-M1 depending on the use of recommended or alternative electroencephalogram (EEG) and electrooculogram (EOG) combinations.
Although the now unified rule for the rating hypopneas and the possibility to detect apneas or hypopneas using one method seem to be a great simplification for clinical routine, other recommendations must be proven in practice. With the publication of version 2.0 of the AASM manual, it must be asked whether the procedures of the manual should be mandatory for use in Germany or if at least specific basic principles could also be used with older PSG equipment. From the scientific position, the use of the AASM manual version 2.0 for sleep research is indespensible.

Keywords Sleep · Breathing · Scoring Methods . Polysomnography · Equipment
$2 \mathrm{~Hz},>75 \mu \mathrm{V}$ ) pro Epoche N3 vorhanden sein müssen.

\section{„Body movement"}

Epochen mit größerer Körperbewegung („body movement“) über mehr als $15 \mathrm{~s}$, ohne dass der EEG-Grundrhythmus der Epoche bestimmt werden kann, erhalten dasselbe Stadium wie die jeweils nachfolgende Epoche. Sofern für einen Teil der
Epoche ein $\alpha$-Rhythmus erkennbar ist, wird die Epoche zu Wach (W). Dies gilt auch, wenn die Dauer des $\alpha$-Rhythmus geringer als $15 \mathrm{~s}$ ist. In Version 2.0 des AASMManual wurde in diesem Zusammenhang ergänzt, dass auch dann $W$ zu geben ist, wenn die Epoche selbst keinen $\alpha$-Rhythmus enthält, aber entweder die Epoche zuvor oder danach $W$ ist.

\section{Arousal-Rating}

Wie bereits im R\&K-Manual beschrieben spielen kurze EEG-Beschleunigungen $(\theta-$, $a$-Wellen und/oder mehr als $16 \mathrm{~Hz})$ über mindestens $3 \mathrm{~s}$ mit und ohne gleichzeitiger EMG-Erhöhung, sog. Arousal, eine wichtige Rolle für die Schlafstadienbestimmung. Im Wesentlichen folgt das AASMManual damit den im Jahr 1992 aufgestellten Kriterien der American Sleep Disor- 
ders Association (ASDA) [4]. Eine maximale Dauer wird nicht definiert, jedoch sollen dem Arousal mindestens $10 \mathrm{~s}$ stabilen Schlafs vorausgehen. Während in allen Non-REM-Stadien (N1-3) das Schlafstadium sich ggf. nach einem Arousal auch ohne EMG-Erhöhung ändern kann, gilt dies nicht für REM. Für einen Stadienwechsel aus REM muss eine größere Körperbewegung („body movement“) erfolgen; ein Arousal - auch mit parallelem EMG-Anstieg - reicht in REM nicht aus. Wie zuvor gilt prinzipiell, dass ein Arousal in der ersten Hälfte der Epoche zu einem Schlafstadienwechsel führen kann. Ein Arousal in der zweiten Epochenhälfte kann zu einer Neubewertung der nachfolgenden Epoche führen. Ausnahmen gelten für REM. Dies gilt insbesondere für die Beachtung, dass Arousal in REM nicht nur die EEG-Frequenzbeschleunigung sondern auch eine EMG-Erhöhung über mindestens eine Sekunde beinhalten müssen. In Version 2.0 wird in einer Fußnote festgelegt, dass Arousal auch im Wachen gewertet und in den Arousalindex eingehen sollen, sofern diese Arousal in der Zeit zwischen Licht-aus und Licht-an auftreten.

\section{Graphoelemente}

Neue Graphoelemente wurden weder in der ersten Version noch in Version 2.0 eingeführt, jedoch wurden schon in der ersten AASM-Manualversion Schlafspindeln auf einen Frequenzbereich von 11$16 \mathrm{~Hz}$ (nach Rechtschaffen und Kales: 12$14 \mathrm{~Hz}$ ) erweitert. Die Definition der anderen Graphoelemente wird dagegen im Wesentlichen nicht verändert. Es wird jedoch klar festgelegt, welche Kanäle zur Erkennung - entsprechend der jeweils maximalen Ausprägung - benutzt werden sollen. Gleichzeitig wird betont, dass die Elemente deutlich von der EEG-Hintergrundaktivität abgrenzbar sein sollen.

\section{Stadium Wach}

Bezüglich des Stadiums W wurde im Jahr 2007 eine grundlegende Änderung der Schlafstadienbestimmung vorgenommen. Obwohl bereits Rechtschaffen und Kales eine okzipitale Erfassung vorschlugen, wurde bis zum Jahr 2007 zumindest bei rein klinischen Fragestellungen das Stadium W anhand von C3 bzw. C4 bestimmt. Dem Manual folgend, soll die Erfassung mittels der okzipitalen Ableitung erfolgen, da sich der a-Rhythmus als grundlegendes Kennzeichen des Wachens hier am besten zeigt. Nach wie vor wird W nur dann gewertet, wenn mindestens 50\% einer Epoche aus $\alpha$-Wellen $(8-13 \mathrm{~Hz})$ oder schnelleren bestehen.

Das Stadium liegt in der Normvariante bei Schläfern ohne erkennbaren okzipitalen $\alpha$-Rhythmus im sicheren $\mathrm{W}$ auch bei Lidschlägen mit geschlossenen oder offenen Augen (zusammengehörende vertikale Augenbewegungen von $0,5-2 \mathrm{~Hz}$ ) vor oder im lesenden Zustand (Serien von Augenbewegungen mit jeweils einer langsamen Phase, gefolgt von einer schnellen Augenbewegung in Gegenrichtung) oder bei unregelmäßigen zusammengehörenden Augenbewegungen bei normalem oder hohem Muskeltonus im Kinn-EMG.

In einer Fußnote wird in Version 2.0 festgelegt, dass die Zeiten bei denen der $\mathrm{Pa}$ tient nicht an das PSG-Gerät angeschlossen ist, als W gewertet werden sollen; ggf. gehen kurze Zeiten in denen währenddessen - wenn überhaupt - Schlaf auftritt nicht in das Scoring ein.

\section{Stadium N 1}

Wie durch Rechtschaffen und Kales beschrieben wird von einem niederamplitudigen gemischtfrequenten EEG gesprochen, wobei eine Aktivität im Bereich von $4-7 \mathrm{~Hz}$ vorherrschen soll. Stadium N1 wird gewertet, wenn der a-Rhythmus abgeschwächt und durch die niederamplitudige gemischtfrequente Aktivität in mindestens 50\% der Epoche ersetzt wird. Vertexzacken und langsame Augenbewegungen gelten weiterhin als Kennzeichen für N1, sind jedoch nicht obligat. In der Normvariante „kein okzipitales $\alpha$ in W“ wird N1 gewertet, sobald bei gleichzeitiger Verlangsamung der Hintergrundaktivität um wenigstens $1 \mathrm{~Hz}$ im Vergleich zu W die EEG-Aktivität bei 4-7 Hz liegt, oder Vertexzacken bzw. langsame Augenbewegungen auftreten.

\section{Stadium N 2}

Wie zuvor gelten die Schlafspindeln und K-Komplexe ohne assoziiertes Arousal als wesentliche Zeichen für Stadium N2. Präzisierend wird in Version 2.0 festgelegt, dass N1 bei Vorliegen von K-Komplexen mit Arousal solange gewertet werden soll, bis K-Komplexe ohne assoziiertes Arousal auftreten. Wie von Rechtschaffen und Kales empfohlen richtet sich der Beginn der jeweils ersten Epoche N2 nach dem Auftreten einer Schlafspindel und/ oder eines K-Komplexes. Tritt diese Ereignis in der ersten Hälfte auf, dann wird diese Epoche bereits als N2 gewertet. Bei Auftreten in der zweiten Hälfte der Epoche wird erst die nachfolgende Epoche als N2 angesehen. Anders als von Rechtschaffen und Kales eingeführt wird N2 fortlaufend gegeben, solange eine niederamplitudige und gemischtfrequente EEG-Hintergrundaktivität vorhanden ist und zuvor eine Spindel und/oder ein K-Komplex vorhanden war. Hinsichtlich des Endes von N2 ist damit das 3-Minuten-Kriterium aufgehoben. Das Ende wird durch - den Übergang in W, N3 oder R oder

- ein Arousal mit und ohne zeitlichen Zusammenhang mit einem K-Komplex terminiert. Es soll ein Übergang zum Stadium N1 erfolgen, solange bis erneut eine Spindel oder ein KKomplex ohne nachfolgendes Arousal erscheint. Im Vergleich zur ersten AASM-Manualversion wird in Version 2.0 in einer Fußnote dargelegt, dass K-Komplexe mit arousalassoziierten Frequenzbeschleunigungen zwar typischerweise eher zentral oder okzipital auftreten, jedoch auch berücksichtigt werden sollen, sofern diese Ereignisse nur in der frontalen Ableitung auftreten.

- Das Ende von N2 wird durch das Auftreten einer größeren Körperbewegung („body movement“) gefolgt von langsamen Augenbewegungen terminiert; sofern keine langsamen Augenbewegungen auftreten wird N2 fortgeführt.

\section{Stadium N 3}

Wie bereits beschrieben werden die ehemaligen Stadien S3 und S4 in Stadium N3 zusammengefasst. Zudem wurde der Literatur entsprechend eine Untergrenze der tiefschlafrelevanten $\delta$-Wellen $(0,5 \mathrm{~Hz})$ eingeführt. Weiterhin müssen aber min- 
destens $20 \%$ der Epoche aus diesen $\delta$ Wellen $(0,5-2 \mathrm{~Hz},>75 \mu \mathrm{V})$ bestehen. Das Amplitudenkriterium gilt für alle Altersgruppen. Seit dem Jahr 2007 besteht die Anweisung, diese Wellen in der frontalen Ableitung zu messen, da sie hier ihre maximale Ausprägung haben. In Version 2.0 wird ergänzend klargestellt, dass K-Komplexe, die das Amplituden- und Frequenzkriterium erreichen, als $\delta$-Wellen gelten. In Version 2.0 wird in einer Fußnote auch dargelegt, dass mit der alternativen $A b$ leitung $\mathrm{Fz}$-Cz keine adäquate frontale $\delta$ Aktivität ermittelt werden kann. Es werden daher in Abhängigkeit von der Kombination empfohlener und alternativer EEGund EOG-Ableitungen die jeweils notwendigen Verschaltungen (u. a. C4-M1) vorgegeben.

\section{Stadium REM (R)}

Wie durch Rechtschaffen und Kales beschrieben sind ein niederamplitudiges gemischtfrequentes EEG, niedriger EMGTonus und schnelle Augenbewegungen (<500 ms) die Grundvoraussetzungen für das Scoren von Stadium R. Als Hilfskriterium gilt, dass die $\alpha$-Aktivität oft $1-2 \mathrm{~Hz}$ niedriger als im Wachen ist sowie das Auftreten von Sägezahnwellen und einer transienten Muskelaktivität. Der Intention von Rechtschaffen und Kales folgend werden der Übergang von N2 in R sowie das Ende von REM definiert. Es ist erneut entscheidend, ob das Auftreten von KKomplexen/Spindeln oder der EMG-Abfall in der ersten oder zweiten Epochenhälfte erfolgt. Treten die Graphoelemente des N2 gemeinsam mit schnellen Augenbewegungen in einer Epoche auf, so soll REM gewertet werden. Das Ende von Stadium $\mathrm{R}$ ist gegeben, wenn

- ein Übergang in N3 oder W erfolgt,

- der Muskeltonus ansteigt und Zeichen für N1 vorhanden sind,

- ein Arousal oder eine Körperbewe-

gung erfolgt. Sofern das EEG nachfolgend weiter niederamplitudig und gemischtfrequent bleibt, aber langsame Augenbewegungen auftreten, wird N1 gewertet. Ohne langsame Augenbewegungen und bei gleichbleibend niedrigem Muskeltonus bleibt $\mathrm{R}$ trotz des Arousals und auch ohne schnelle Augenbewegungen erhalten.
- Das Ende von Stadium R ist auch gegeben, wenn in der Abwesenheit von schnellen Augenbewegungen mindestens ein K-Komplex bzw. eine Spindel in der ersten Epochenhälfte auftreten (dies gilt auch für einen gleichbleibend niedrigen Muskeltonus). Ergänzend wird in Version 2.0 festgestellt, dass in diesem Fall N2 zu werten ist, sofern nicht $\mathrm{N} 3$ erreicht wird.

Allerdings können gerade in der ersten REM-Phase K-Komplexe oder Spindeln in Epochen auftreten, die ansonsten alle 3 REM-Kriterien (gemischtfrequente niederamplitudige Aktivität, niedriger Muskeltonus und schnelle Augenbewegungen) erfüllen. Sofern schnelle Augenbewegungen in der entsprechenden Epoche auftreten, soll dann R gewertet werden. Des Weiteren wird festgehalten, dass Regeln zum Übergang zwischen N1 und R nicht existieren, sondern $R$ gewertet werden soll, wenn alle 3 REM-Kriterien zutreffen.

\section{Kardiale Parameter}

Hier werden die Platzierung der EKGElektroden sowie die EKG-Auswertungsregeln für Sinustachykardien, Bradykardien, Asystolien usw. für Personen, die älter als 6 Jahre sind, festgelegt ohne dass es hier Änderungen in Version 2.0 gab.

\section{Bewegungsstörungen}

Hinsichtlich der periodischen Beinbewegungen gilt seit dem Jahr 2007, dass der Muskeltonus mindestens $8 \mu \mathrm{V}$ über der Ruheaktivität liegen muss. Entsprechend beginnt die Bewegung an dem Punkt, an dem dieser Anstieg von $8 \mu \mathrm{V}$ erreicht wird, und endet sobald über mindestens 0,5 s der Anstieg nicht mehr als $2 \mu \mathrm{V}$ beträgt. Die Dauer der einzelnen Bewegung liegt zwischen 0,5 und 10 s. Für Serien periodischer Beinbewegungen (PLM) wurden im Wesentlichen die bereits bekannten Regeln nach Allen et al. [2] in das Manual integriert (4 Bewegungen in Folge mit 5-90 sekündigen Abständen zwischen den einzelnen Bewegungen). Finden sich Bewegungen in beiden Beinen, so werden diese auch dann als einzelne Ereignisse innerhalb der Serie gewertet, wenn weniger als $5 \mathrm{~s}$ dazwischen liegen.
Ein PLM ist dann arousalassoziiert, wenn zwischen dem Beginn beider Ereignisse nicht mehr als 0,5 s liegen. Dies gilt unabhängig davon, welches Ereignis zuerst auftritt. Beinbewegungen sollen nicht gewertet werden, wenn sie $0,5 \mathrm{~s}$ vor bis $0,5 \mathrm{~s}$ nach einer Apnoe oder Hypopnoe auftreten. In Version 2.0 wird weiterhin spezifiziert, dass von 2 Beinbewegungen, die weniger als 10 s auseinander liegen und jeweils mit einem Arousal einhergehen, zwar beide als Extremitätenbewegung gewertet werden können, aber nur das erste der beiden Ereignisse als PLM mit Arousal.

Definiert, aber weder verändert, ergänzt und spezifiziert, wurden ebenfalls die Regeln zum Scoren

- alternierender Beinbewegungen,

- des hpnagogen Fußtremors,

- des exzessiven fragmentarischen Myoklonus sowie

- eines Bruxismus und

- der REM-Schlaf-Verhaltensstörung oder

- anderer rhythmischer Bewegungsstörungen.

\section{Atmungsparameter}

Nach einer ausführlichen Analyse der Literatur wurden in der ersten AASM-Manualversion verbindliche Regeln festgelegt, die allerdings in Version 2.0 erneut hinsichtlich der Erfassung von Apnoen und dem Scoring von Hypopnoen modifiziert wurden. Neben Apnoen und Hypopnoen wurden auch Kriterien für sog. RERA, Hypoventilation und die CheyneStokes-Atmung definiert und teils in Version 2.0 verändert. Die Evidenzbasierung und Konsensusfindung hierzu [7] sind ebenso wie eine ausführliche deutsche Übersicht über die notwendige Messtechnik [10] publiziert.

\section{Messmethodik}

In der ersten AASM-Manualversion wurde zur Erfassung von Apnoen ein oronasaler Thermistor empfohlen. Hypopnoen hingegen sollten durch eine nasale Staudruckmessung mit oder ohne Berechnung der Quadratwurzel des Signals erfasst werden (Empfehlung). Sofern diese Methoden nicht funktionieren oder kein verlässliches Signal geben, können gemäß Version 2.0 
Hier steht eine Anzeige.

黛 Springer 
Apnoen alternativ auch mittels Staudrucksensor oder Induktionsplethysmographie (Fluss oder Summe, kalibriert oder nicht kalibriert) und Hyponoen mittels Plethysmographie, oronasalen Thermistors oder dualer Induktionsgurte erfasst werden. Auch ist die Ösophagusmanometrie nicht mehr die zwingende Methode zur Erfassung von RERAs. Es können ebenso duale Induktionsgurte zum Einsatz kommen. Zusätzlich wird nun in Version 2.0 verbindlich festgelegt, dass während einer positiven Drucktiration (PAP-Titration) das Flusssignal des PAP-Geräts zur Identifikation von Apnoen oder Hypopnoen genutzt werden soll.

Die Sauerstoffsättigung wird über die Pulsoximetrie gemessen. Die Signale dürfen über maximal $3 \mathrm{~s}$ gemittelt werden. Ergänzt wird dies in Version $2.0 \mathrm{um}$ die Angabe: bei einer Herzfrequenz von 80 Schlägen pro Minute.

In Version 2.0 wird auch deutlicher dargestellt, dass zur Messung des Schnarchens

- ein akustischer oder

- piezoelektrischer Sensor oder

- ein nasaler Drucksensor

verwendet werden kann. Zur Erfassung von Hypoventilationen kann sowohl eine arterielle, transkutane oder endtidale $\mathrm{pCO}_{2}$-Messung - letztere nicht bei PAPTitrationen - zum Einsatz kommen.

Es bleibt weiter unverändert, dass die Dauer eines Ereignisses vom Nadir des letzten Atemzugs vor der deutlichen Verminderung bis zum Beginn des ersten wieder regulären Atemzugs gemessen wird.

\section{Apnoen}

Folgende Parameter müssen zur Wertung einer Apnoe gegeben sein:

- Verminderung des Signalpeaks um mindestens $90 \%$ des Baselineniveaus,

- Dauer dieser Verminderung über einen Zeitraum von mindestens $10 \mathrm{~s}$.

In der ersten AASM-Manualversion lautete die Definition: Das Ereignis dauert mindestens $10 \mathrm{~s}$ und die Verminderung besteht über mindestens $90 \%$ des Ereignisses. Daraus folgt, dass ein Ereignis von 10-sekündiger Länge mit einer Verminderung auf maximal 10\% des Baseline- niveaus über einer Dauer von $9 \mathrm{~s}$ gefolgt von einer Sekunde Hypopnoe als Apnoe gewertet wurde. Dies ist nun nicht mehr möglich, da die Verminderung gemäß Version 2.0 mindestens $10 \mathrm{~s}$ andauern muss. Ein weiterer entscheidender Unterschied zur ersten Manualversion besteht darin, dass Apnoen gewertet werden sollen, sofern der Beginn oder das Ende der Apnoe in eine Epoche Schlaf fällt. Lediglich Ereignisse, die komplett im Wachen auftreten, gehen nicht in den Index ein, sondern sollen im summierten Bericht deskriptiv berichtet werden.

Die Unterscheidung in

- obstruktive Apnoe (erhaltener oder erhöhter inspiratorischer Effort),

- zentrale Apnoe (ohne inspiratorischen Effort) und

- gemischte Apnoe (fehlender inspiratorischer Effort zu Beginn, gefolgt von Atemanstrengung im 2. Teil des Ereignisses)

bleibt unverändert. Ergänzend wird in Version 2.0 erklärt, dass die einzelnen Zeitverhältnisse bei gemischten Apnoen nicht näher festgelegt werden können.

\section{Hypopnoen}

Im Vergleich zur ersten AASM-Manualversion findet sich hier die wesentlichste Änderung in Version 2.0. In der ersten Version bestand die alternative Regelung, dass entweder eine mindestens 30\%ige Verminderung des nasale Drucks und ein mindestens 4\%iger Abfall der Sauerstoffsättigung oder eine mindestens 50\%ige Verminderung des nasale Drucks und ein mindestens 3\%iger Abfall der Sauerstoffsättigung bzw. das Ereignis arousalassoziiert sein sollte.

Demgegenüber wird in Version 2.0 des AASM-Manuals nur eine Definition festgelegt: Eine Hypopnoe besteht demnach, wenn

- das Flowsignal um mindestens 30\% im Vergleich zum Ausgangswert fällt,

- diese Verminderung mindestens $10 \mathrm{~s}$ andauert (analog zur gemäß Version 2.0 präzisierten Apnoedefinition) und - die Sauerstoffsättigung um wenigstens 3\% im Vergleich zum Ausgangswert fällt oder das Ereignis arousalkorreliert ist.
„Respiratory effort related arousal“

Die Regeln zur Definition einer RERA bleiben in Version 2.0 unverändert. Die Ösophagusmanometrie wird aber nicht mehr als Methode der Wahl genannt. Eine RERA ist eine mindestens 10-sekündige Sequenz von Atemzügen, die durch einen erhöhten Atmungsaufwand oder ein Abflachen („flattening“) der Wellenform des Atemflusses charakterisiert ist und dabei zu einem Arousal führt ohne die Kriterien einer Apnoe oder Hypopnoe zu erfüllen.

\section{Hypoventilation}

Sofern Hypoventilationen gemessen werden sollen, wird in Version 2.0 die Definition erweitert. Während zuvor ein Anstieg des $\mathrm{pCO}_{2}$ um mindestens $10 \mathrm{mmHg}$ gefordert wurde, wird nun spezifiziert, dass der $\mathrm{pCO}_{2}$-Anstieg sich über wenigstens 10 min erstrecken soll. Dabei sollen

— entweder $55 \mathrm{mmHg}$ über mindestens 10 min überschritten werden oder

- ein Anstieg von wenigstens $10 \mathrm{mmHg}$ auf mehr als $50 \mathrm{mmHg}$ über mindestens 10 min vorliegen.

\section{Cheyne-Stokes-Atmung}

Die Definition der Cheyne-Stokes-Atmung wurde in Version 2.0 ebenfalls geändert. Neben den schon bestehenden Kriterien von wenigstens 5 zentralen Apnoen oder Hypopnoen pro Stunde Schlaf wird ergänzt, dass das zyklische Crescendo-Decrescendo des Atmungsmusters mit diesen Apnoen bzw. Hypopnoen assoziiert sein muss. Während zuvor ein Cheyne-Stokes-Zyklus mindestens $10 \mathrm{~min}$ andauern musste, besteht nun der einzelne Zyklus aus wenigstens

- 3 konsekutiven zentralen Apnoen bzw. Hypopnoen,

- die durch das typische CrescendoDecrescendo-Muster voreinander abgrenzbar sind,

- wobei die Gesamtdauer dieses Zyklus mindestens $40 \mathrm{~s}$ beträgt.

Insgesamt muss eine Cheyne-Stokes-Atmung mit mehr als 5 zentralen Apnoen bzw. Hypopnoen pro Stunde Schlaf über mehr als $2 \mathrm{~h}$ des Monitorings vorliegen. 


\section{Zusammenfassung und Ausblick}

Schon die erste Version des AASM-Manuals [13] basierte auf einer im Vorfeld vorgenommenen evidenzbasierten Durchsicht der jeweiligen Literatur. Es wurden so für die Bereiche technische Spezifikationen, kardiale Störungen, Bewegungsstörungen und Atmungsparameter die jeweiligen Kriterien der Messung und Auswertung, die sich in vielen Laboren - auch in Deutschland - bereits als Standard etabliert hatten, vereinheitlicht. Im Vergleich zum R\&K-Manual [14] enthält der Teil der Schlafstadienbestimmung wesentliche Neuerungen, wobei jedoch stets die Intention des R\&K-Manuals gewahrt blieb. In weiten Teilen wurden lediglich mögliche bestehende Unklarheiten ausgeräumt. Neben der Neubenennung der Stadien war allerdings neu: die Zusammenfassung der Tiefschlafstadien in N3, das Werten von W anhand des okzipitalen EEGs bzw. das Werten der N3-relevanten $\delta$-Wellen anhand des frontalen EEGs sowie einige Regeln zur Fortführung der Stadien bzw. zum Schlafstadienwechsel.

Obwohl insgesamt viele Einzelheiten präzisiert wurden, gehen die Definitionen der Graphoelemente [16] - gerade für Anfänger - auch in der revidierten AASMManualfassung nicht weit genug über das altbekannte R\&K-Manual hinaus.

Im Vergleich zur ersten Version wurden in Version 2.0 des AASM-Manuals [7] im Bereich der Schlafstadienwertung nur einige kleine ergänzende Präzisierungen vorgenommen. Als wesentlich ist aber die komplette Überarbeitung der Regeln für die Messung und Auswertung der Atmung zu nennen. Obwohl ein paralleles Anlegen und Messen von oronasalem Thermistor und Staudrucksonde durchaus möglich ist, stellt die gemäß Version 2.0 mögliche pragmatische Erfassung sowohl von Apnoen als auch von Hypopnoen über eine gemeinsame Methode für viele Labore eine deutliche Erleichterung dar. Insbesondere die ursprünglich alternative Regelung bzgl. der Hypopnoemessung war eher verwirrend als nützlich [11]. Nun ist wieder eine weltweit einheitliche Auswertung möglich. Auch wurden viele Details präzisiert oder neu gefasst. Dies betrifft v. a. die Apnoedefinition mit einer Dauer der Flowvermin- derung über $10 \mathrm{~s}$ und nicht nur - wie zuvor festgelegt - über $90 \%$ einer Dauer von wenigstens $10 \mathrm{~s}$. Bemerkenswert ist auch, dass die Definition der Cheyne-Stokes-Atmung geändert und die Definition der Hypoventilationen präzisiert wurde. Letztlich muss sich trotz der genannten Erleichterung erst noch zeigen, ob sich die neuen Festlegungen bezüglich der Dauer der Flowverminderung, der engeren Definition von Hypoventilationen und der Cheyne-Stokes-Atmung in der täglichen Arbeit mit den Patienten durchsetzen werden. Dies gilt umso mehr als dass die entsprechenden Definitionen nicht denen der International Classification of Sleep Disorders (ICSD-2, [3]) entsprechen.

\section{Übernahme in die klinische Routine}

Insgesamt scheint es, dass neben der evidenzbasierten Betrachtung der einzelnen Methoden auch ihre klinische Anwendung bei Patienten vermehrt Eingang gefunden hat, sodass eine größere Akzeptanz des AASM-Manuals immer wahrscheinlicher wird. In den USA ist die Übergangsfrist zur Umsetzung bereits im Jahr 2008 abgelaufen. Demgegenüber muss für Deutschland festgestellt werden, dass noch immer viele Labore mit PSG-Geräten arbeiten müssen, die eine komplette Umsetzung des AASMManuals insbesondere hinsichtlich der Durchführung

- eines 3- oder gar 6-Kanal-EEGs,

- eines peripheren 2-Kanal-EMGs,

- einer beidseitigen EMG-Ableitung des M. tibialis anterior in 2 Kanälen oder

- der parallelen Erfassung des Atemflusses mit Thermistor und Staudruckmessung

nicht erlauben. Diese Feststellung bezieht sich auf Daten aus der Qualitätssicherungsrunde der deutschen Gesellschaft für Schlafmedizin (DGSM) aus dem Jahr 2012. Gleichzeitig stellt sich mit Version 2.0 auch die Frage, welche Änderungen in technisch-apparativer Hinsicht notwendigerweise im klinischen Alltag inhaltlich umgesetzt werden müssen. Für wissenschaftliche Fragestellungen ist die Umsetzung des AASM-Manuals eine Selbstverständlichkeit.
Bezüglich der EEG-Parameter sind alle PSG-Geräte in der Lage, mindestens 2 EEGs aufzuzeichnen. Dabei ist es ohne Schwierigkeit möglich, davon ein EEG okzipital zu platzieren, sodass zumindest der $a$-Rhythmus im ruhigen Wach besser erfasst werden könnte. Da die Erfassung tiefschlafrelevanter $\delta$-Wellen gemä $\beta$ AASM-Manual, Version 2.0, quasi als Notlösung auch mittels C3/M2 respektive C4/M1 erfolgen kann, stellt sich aber die Frage, ob evtl. auf die technische Voraussetzung zum Abgreifen eines dritten frontal platzierten EEGs sowie auf sog. Backup-Kanäle verzichtet werden kann. Dies gilt umso mehr, da

- sich im Vergleich von Schlafstadienanalysen gemäß AASM-Manual versus R\&K-Kriterien die Interraterreliabilität nur minimal verbessert hat (Übersicht in [11]),

- sich je nach Untersuchung Veränderung der prozentualen Schlafanteile eher auf N1 oder N2 bei übereinstimmender Zunahme von N3 beziehen $[11,18]$ und

- AASM-bezogene Alters- und Geschlechtsnormwerte noch fehlen.

Ähnliches gilt für das submentale EMG, wobei die Elektrodenplatzierung submental gegen mental ebenfalls sofort in allen Laboren umgesetzt werden könnte. Der zweite entsprechende EMG-Kanal ist als Back-up-Kanal nur dienlich, wenn sich die Signalqualität des submentalen EMGs verschlechtert, nicht aber wenn sich das mentale EMG als Referenz für beide submentalen Elektroden löst. Hinsichtlich der Erfassung und Auswertung der peripheren EMGs zur beidseitigen Ableitung des M. tibialis anterior hat sich in Version 2.0 praktisch nichts geändert. Die Umsetzung von einer manuellen auf eine computerisierte Erfassung der Beinbewegungen anhand des definierten Anstiegs und Abfall der EMG-Spannung dürfte dagegen auch für die meisten älteren Geräte konfigurierbar sein, zumal die kombinierte Ableitung beider Bein-EMGs auf nur einem Kanal möglich ist. Dies wird zwar nicht präferiert, aber für klinische Fragestellungen gemäß einer Fußnote erlaubt. Aus klinischer Sicht ist dagegen kritischer zu bewerten, dass Beinbewegungen, die in engem zeitlichen Zusammen- 
hang $( \pm 0,5 \mathrm{~s})$ mit einer Apnoe oder Hypopnoe stehen, immer noch nicht als solche gewertet werden sollen und dementsprechend nicht in den Index eingehen. Dementsprechend kann im klinischen Alltag derzeit nur beschreibend eine Aussage zu Beinbewegungen im Zusammenhang mit Atmungsstörungen getroffen werden, obwohl sich gerade bei Patienten mit schlafbezogenen Atmungsstörungen eine hohe Assoziation zum Syndrom periodischer Beinbewegungen findet $[1,8]$. Gerade aber auch die Periodizität von Beinbewegungen wird durch das Auftreten von Apnoen bzw. Hypopnoen maskiert und kann sich - in Abhängigkeit vom Schweregrad der schlafbezogenen Atmungsstörung - unter einer PAP-Therapie verbessern oder verschlechtern [5]. Durch die Einführung eines entsprechenden gesonderten Index könnte nicht nur der klinische Blick auf diese Problematik gestärkt werden. Dem weiterbehandelnden Arzt könnte auch leichter als durch die rein klinische Beschreibung vermittelt werden, dass hier ggf. ein gesonderter Therapiebedarf besteht. Wie bereits erwähnt bleibt hinsichtlich der neu gestellten Definitionen für die Cheyne-Stokes Atmung und die Hypoventilationen in Abweichung zu ICSD-2 die Umsetzung im klinischen Alltag abzuwarten.

\section{Fazit für die Praxis}

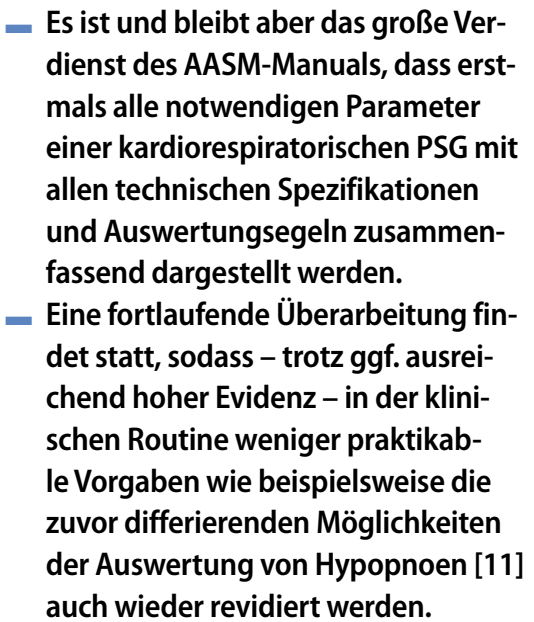

\section{Korrespondenzadresse}

Prof. Dr. A. Rodenbeck

CharitéCrossOver (CCO),

Institut für Physiologie ,

Charité-Universitätsmedizin Berlin

Charitéplatz 1, 10117 Berlin

arodenb@gwdg.de

Interessenkonflikt. Die Autorin gibt an, dass kein Interessenkonflikt besteht.

\section{Literatur}

1. Al-Alawi A, Mulgrew A, Tench E, Ryan CF (2006) Prevalence, risk factors and impact on daytime sleepiness and hypertension of periodic leg movements with arousals in patients with obstructive sleep apnea. J Clin Sleep Med 2:281-287

2. Allen RP, Picchietti D, Hening WA et al (2003) Restless legs syndrome: diagnostic criteria, special considerations, and epidemiology. A report from the restless legs syndrome diagnosis and epidemiology workshop at the National Institutes of Health. Sleep Med 4:101-119

3. American Academy of Sleep Medicine (2005) ICSD-2, International classification of sleep disorders, 2. Aufl. Diagnostic and coding manual. American Academy of Sleep Medicine, Westchester

4. American Sleep Disorder Association ASDA (1992) EEG arousals: scoring rules and examples. Sleep 15:173-184

5. Baran AS, Richert AC, Douglass AB et al (2003) Change in periodic limb movement index during treatment of obstructive sleep apnea with continuous positive airway pressure. Sleep 26:717-720

6. Berry RB, Brooks R, Gamaldo CE et al (2012) The AASM Manual for the scoring of sleep and associated events: rules, terminology and technical specifications. Version 2.0. http://www.aasmnet.org, Darien, Illinois, American Academy of Sleep Medicine

7. Berry RB, Budhiraja R, Gottlieb DJ et al (2012) Rules for scoring respiratory events in sleep: update of the 2007 AASM manual for the scoring of sleep and associated events. Deliberations of the sleep apnea definitions task force of the American Academy of Sleep Medicine. J Clin Sleep Med 8:597-619

8. Chervin RD (2001) Periodic leg movements and sleepiness in patients evaluated for sleep-disordered breathing. Am J Respir Crit Care Med 164:14541458

9. Fitch K, Bernstein SJ, Aguilar MD et al (2001) The RAND/UCLA Appropriateness method user's manual. RAND Corporation, Santa Monica

10. Glos M, Penzel T (in Druck) Diagnostik von schlafbezogenen Atmungsstörungen. In: Schulz H, Geisler P, Rodenbeck A (Hrsg) Kompendium Schlafmedizin, 21. Ergänzungslieferung, Kapitel I-4.11.2. ecomed MEDIZIN, Heidelberg

11. Grigg-Damberger MM (2012) The AASM scoring manual four years later. J Clin Sleep Med 8:323-332

12. Iber $C$ et al (2007) The new sleep scoring manual. J Clin Sleep Med 3:107-204
13. Iber C, Ancoli-Israel S, Chesson A, Quan SF for the American Academy of Sleep Medicine (2007) The AASM manual for the scoring of sleep and associated events: rules, terminology and technical specifications, 1st ed. Westchester, Illinois, American Academy of Sleep Medicine. Deutsche Übersetzung: Das AASM Manual zum Scoring von Schlaf und assoziierten Ereignissen: Regeln, Terminologie und technische Spezifikationen. Steinkopff/Springer, Berlin

14. Rechtschaffen A, Kales A (Hrsg) (1968) A manual of standardized terminology, techniques, and scoring system for sleep stages of human subjects, Washington Public Health Service, US Government Printing Office, Washington. Deutsche Übersetzung: Ein Manual der standardisierten Terminologie, Techniken und Auswertung der Schlafstadien beim Menschen. ecomed, Landsberg/Lech. 2002

15. Rodenbeck A (in Druck) Auswerteregeln der American Academy of Sleep Medicine - Version 2.0. In: Schulz H, Geisler P, Rodenbeck A (Hrsg) Kompendium Schlafmedizin, 21. Ergänzungslieferung, Kapitel I-9.3.2.1. ecomed MEDIZIN, Heidelberg

16. Rodenbeck A, Binder R, Geisler P et al (Task Force "Scoring of Polysomnographic Recordings" of the German Sleep Society [DGSM]) (2006) A review of sleep EEG patterns. Part 1: a compilation of amended rules for their visual recognition according to Rechtschaffen and Kales. Somnologie 10:159-175

17. Rodenbeck A, Danker-Hopfe H, Penzel T (2008) Auswerteregeln der American Academy of Sleep Medicine (AASM Manual 2007). In: Schulz H, Geisler P, Rodenbeck A (Hrsg) Kompendium Schlafmedizin, 12. Ergänzungslieferung, Kapitel I-9.3.2. ecomed MEDIZIN, Heidelberg

18. Rosenberg RS, Van Hout S (2013) The American Academy of Sleep Medicine inter-scorer reliability program: sleep stage scoring. J Clin Sleep Med 9:81-87

19. Schulz H (2010) Die geschichtliche Entwicklung der Schlafforschung in Berlin. Ein historischer Streifzug Teil I. Somnologie 14:140-156

20. Schulz H (2010) Die geschichtliche Entwicklung der Schlafforschung in Berlin. Ein historischer Streifzug Teil II. Somnologie 14:221-233

21. Schulz H (2011) Die Entwicklung der Schlafstadienklassifikation. In: Schulz H, Geisler P, Rodenbeck A (Hrsg) Kompendium Schlafmedizin, 17. Ergänzungslieferung, Kapitel I-9.3.1. ecomed MEDIZIN, Heidelberg 